\title{
Apparent Diffusion Coefficient Value as a Predictive Marker to Evaluate Tumor Response in Patients with Cervical Cancer
}

\author{
Fariba Allahmoradi, ${ }^{1,}$ Abbas Haghparast, ${ }^{1}$ and Salman Zakariaee ${ }^{2}$ \\ ${ }^{1}$ Kermanshah University of Medical Sciences, Kermanshah, Iran \\ ${ }^{2}$ Tehran University of Medical Sciences, Tehran, Iran \\ "Corresponding author: Fariba Allahmoradi. E-mail: fariba.am93@gmail.com
}

Received 2016 December 21; Accepted 2017 February 08.

\begin{abstract}
Background: Cervical cancer is the second common cancer and the third reason of mortality among women in the world which is often occurred in third and fifth decades of their life. Cervical tumor recurrence observed in $30 \%$ of the patients and the 5 -years survival rate of cervical cancer is about \%50. The development of a rapid and non-invasive method for treatment response evaluation is one of the most challenges in cancer treatment management.Diffusion-weighted magnetic resonance imaging (DW-MRI) is a contrast agent free imaging technique thatis widely used for oncology studies.

Objectives: In this paper, thecapability of ADC valueto monitor the treatmentresponse in the cervical cancer patientswasevaluated. Methods: A computerized search using the databases SID, Google Scholars, Pub med and Science Direct, covering the period from 1998 to 2015, was conducted using the following key search terms: "Treatment response", "Diffusion-weighted magnetic resonance imaging", "ADC”, “MRI” and “Cervical Cancer". In total, 27 relevant papers were reviewed.

Results: Studies had shown thatthe mean ADC value of cervical carcinoma $\left(1.110 \pm 0.175 \times 10^{-3} \mathrm{~mm}^{2} / \mathrm{s}\right)$ is significantly lower than that of normal cervical tissue $\left(1.593 \pm 0.151 \times 10^{-3} \mathrm{~mm}^{2} / \mathrm{s}\right)(\mathrm{P}<0.001)$. The mean ADC value of the cervical area significantly increased after chemoradiationtreatment $\left(1.436 \pm 0.129 \times 10^{-3} \mathrm{~mm}^{2} / \mathrm{s}\right)$ than that before therapy $\left(1.013 \pm 0.094 \times 10^{-3} \mathrm{~mm}^{2} / \mathrm{s}\right)(\mathrm{P}<0.001)$. There is a statistically significant difference between the ADC values of the normal cervical tissue and cervical area after therapy $(\mathrm{P}<0.01)$. Partial response of the bulky tumorscould be predicted with high sensitivity (85.7\%) and specificity (100\%) (AUC 0.96; $\mathrm{P}=0.05$ ) using the ADC value greater than $0.95 \times 10^{-3} \mathrm{~mm}^{2} / \mathrm{s}$.

Conclusions: ADC valuecould be a prognostic factor to monitor the response to therapy for patients with cervical cancer. The addition of DW-MRI to the conventional MRI protocolsconsiderably improved the diagnostic ability of MR imaging method for tumor evaluations.
\end{abstract}

Keywords: Treatment Response, Diffusion-Weighted Magnetic Resonance Imaging, DW-MRI, ADC, Cervical Cancer

This is an abstract presented in the 33rd Iranian congress of radiology (ICR) and the 15th congress of Iranian radiographic science association (IRSA). 